\title{
Electronics and Calibration system for the CMS Beam Halo Monitor
}

Nicolò TOSI*

Università e INFN Bologna (IT)

E-mail: nicolo.tosi@cern.ch

\section{A. E. Dabrowski, R.Loos, S. Orfanelli}

CERN

F. Fabbri, A. Montanari

INFN Bologna

\section{A. Finkel, R. Rusack}

University of Minnesota

\section{P. Stickland}

Princeton University

In the context of increasing luminosity of LHC, it will be important to accurately measure the Machine Induced Background. A new monitoring system will be installed in the cavern of the Compact Muon Solenoid (CMS) experiment for measuring the beam background at high radius. This detector is composed of synthetic quartz Cherenkov radiators, coupled to fast photomultiplier tubes (PMT). The readout chain of this detector will make use of many components developed for the Phase 1 upgrade to the CMS Hadron Calorimeter electronics, with a dedicated firmware and readout adapted to the beam monitoring requirements. The PMT signal will be digitized by a charge integrating ASIC (QIE10), providing both the signal rise time and the charge integrated over one bunch crossing. The backend electronics will record bunch-by-bunch histograms, which will be published to CMS and the LHC using the newly designed CMS beam instrumentation specific DAQ. A calibration monitoring system has been designed to generate triggered pulses of light to monitor the efficiency of the system.

Technology and Instrumentation in Particle Physics 2014

2-6 June, 2014

Amsterdam, the Netherlands

\footnotetext{
* Speaker.
} 


\section{Introduction}

A new monitoring system will be installed in the cavern of the Compact Muon Solenoid (CMS) experiment for measuring the beam induced background at relatively high radius [1]. This system, called Beam Halo Monitor (BHM), will be composed of forty identical units. Each unit will feature a synthetic quartz Cherenkov radiator, directly coupled to a fast, UV sensitive, photomultiplier tubes (PMT).

In order to discriminate machine induced background from proton-proton ( $p p$ ) collision products, BHM will make use of both the signal amplitude (from the directionality of Cherenkov light) and the timing information (exploiting the detector placement along the z-axis of CMS). In the next section the electronics for BHM will be presented, followed by measurements performed at a test beam. The calibration and monitoring system will be discussed in the last section.

\section{Electronics}

The readout chain of BHM will make use of many components developed for the Phase 1 upgrade to the CMS Hadron Forward (HF) calorimeter electronics [2].

These components are subdivided into a fully custom, radiation hard Front-End and a $\mu$ TCA based Back-End, connected by optical links, according to the general scheme in Fig. 1

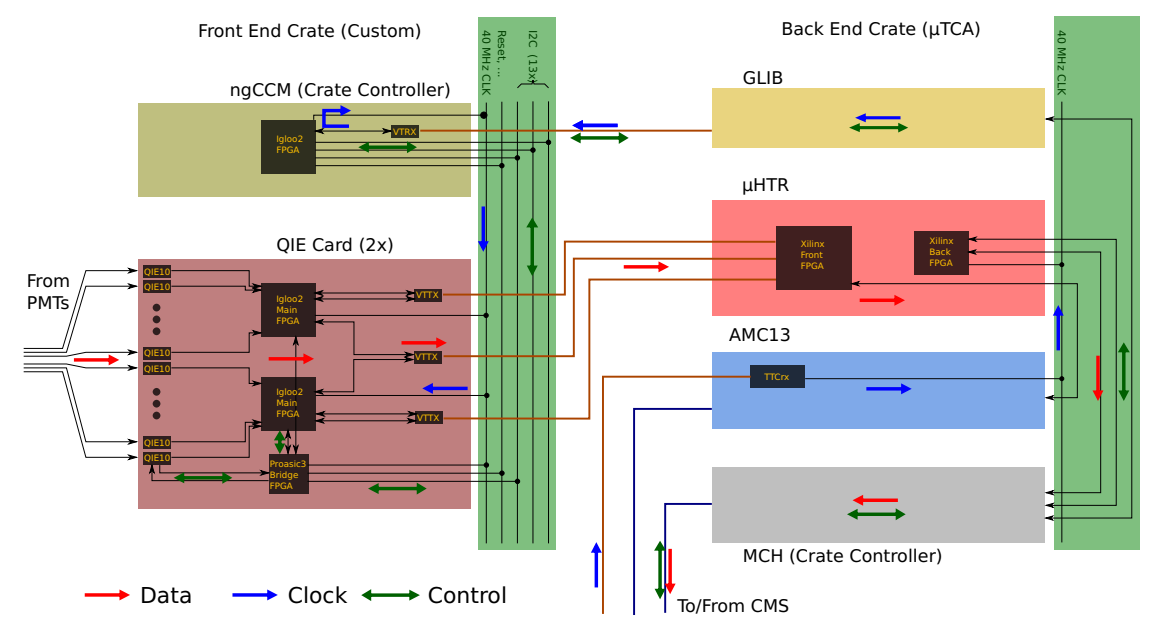

Figure 1: Overview of the BHM Electronics

\subsection{Front-End electronics}

The main components of the Front-End part of the system are:

- QIE10 ASIC, a high dynamic range charge integrating ADC and TDC. It ensures deadtimeless readout of signal amplitude and edge time information (500 ps resolution).

- QIE card, with 24 QIE channels, IGLOO2 FPGAs for data formatting and serialization at 5.0 Gbps; optical links with CERN VTTx [3].

- ngCCM card, the front end crate controller. Manages the CMS clock distribution and slow control through I2C and JTAG busses. 


\subsection{Back-End electronics}

The Back-End is a commercial $\mu$ TCA crate with a standard MCH (controller hub). The readout and slow control will make use of standard Gigabit Ethernet links. The crate houses the following components:

- $\mu$ HTR card, features two Virtex 6 FPGAs and 24 optical link receivers. Handles data decoding, performs signal discrimination based on amplitude and timing cuts, stores individual channel bunch-by-bunch histograms, optionally provides trigger signals.

- AMC13 card, handles clock and TTC distribution to the Back-End crate.

- GLIB card, provides clock and slow control commands to the ngCCM through an optical link.

In order to be used in BHM, some small modifications to these components are required, such as an increase in the capacity to generate and store histograms and the possibility of operating during beam ramp (when the LHC clock frequency is changing). The firmware modifications necessary to implement these extra requirements are currently under development.

\section{Test Beam Results}

The HCAL readout electronics has been tested at the Fermilab Test Beam Facility in April 2014. The main purpose of the test beam was to assess the readiness of the various components and begin to evaluate their performance. A prototype BHM detector unit was connected to the HF QIE card using a cabling scheme similar to that proposed for the actual detector.

Electrical noise on the baseline, as well as PMT dark noise, were measured (Fig. 2a) using the newly commisioned electronics. Electrical noise pickup from the $100 \mathrm{~m}$ signal cable is within the design constraints ( $« 1$ p.e. charge). PMT dark noise is visible in the form of the single p.e. peak, at an amplitude of about $50 \mathrm{fC}$ above baseline. The discontinuities in the histograms are artifacts due to the transitions between QIE ranges and subranges, resulting in different bin widths.

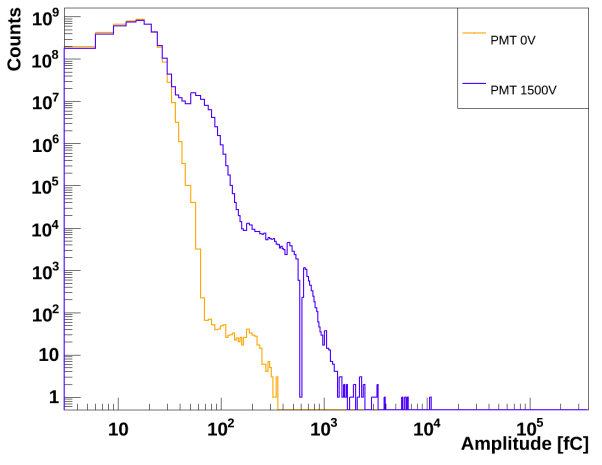

(a) Pedestal and PMT dark noise

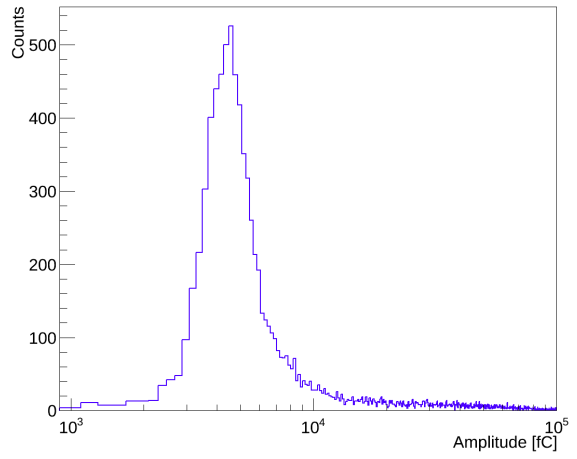

(b) Signal from PMT

Figure 2

The signal from the BHM PMT, in coincidence with the beam trigger, was successfully acquired (Fig. 2b); this allowed to verify the functionality of the data acquisition chain. Most of the functionality related to the slow control was also tested successfully. 


\section{Calibration system}

A calibration and monitoring system (Fig. 3) is foreseen for BHM, to evaluate the performance variations in the PMTs and the Cherenkov radiators. The system will use a light signal produced by UV emitting LEDs and driven to each detector unit through quartz optical fibers and splitters. Splitters will be composed of a fiber bundle coupled with a mirror. The light entering from one of the fiber will be reflected into the others. The light will be injected on the front of the quartz bar, and the resulting PMT signal will be compared with that of a reference photodetector that receives the same light signal (through the splitter). The LED Pulser circuit will be housed on a mezzanine card mounted on the QIE front-end card, while the reference photodetectors will be read out by spare input channels. The calibration and monitoring system will also see heavy use during the commissioning of BHM, assisting with the tuning of the PMT bias and the signal timing.

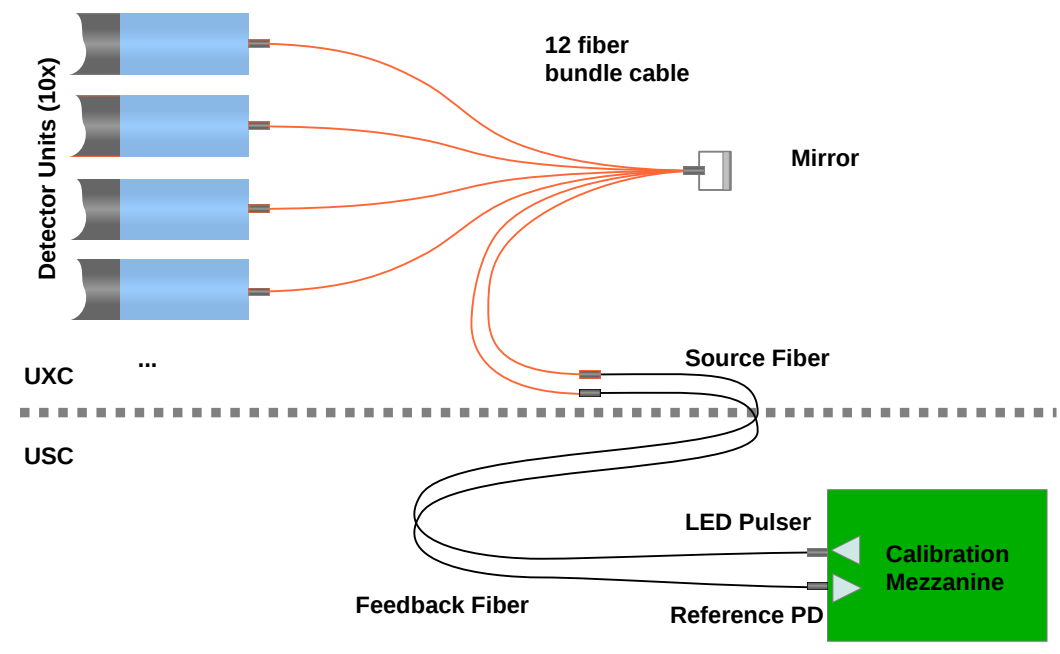

Figure 3: Overview of the calibration system

\section{Summary and outlook}

The test beam results have demonstrated the correct functioning of the individual components of the electronics. Minor issues with the first prototypes have been identified and will be fixed in the preproduction version. Work is ongoing on the integration of the complete system. The calibration system design is completed and its construction has begun; this system will be an extremely useful tool during the commissioning of BHM.

\section{References}

[1] S. Orfanelli et al. Design of a Novel Cherenkov Detector System for Machine Induced Background Monitoring in the CMS Cavern Proceedings IBIC2013

[2] J. Mans et al. CMS Technical Design Report for the Phase 1 Upgrade of the Hadron Calorimeter CERN-LHCC-2012-015/CMS-TDR-010

[3] F. Vasey et al. The Verstile Link common project: feasibility report JINST 7 - 2012 